\title{
Maintenance therapy for patients with advanced non-small cell lung cancer: fact or myth?
}

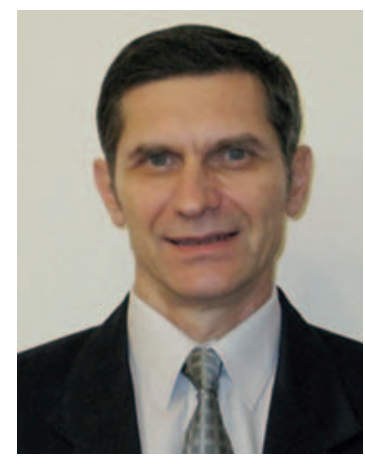

Mircea Dediu, MD, PhD

M. Dediu

Medical Oncology Department, Institute of Oncology “Alexandru Trestioreanu”, Bucharest, Romania

Received 4 February 2011; accepted 10 February 2011

Maintenance therapy (MT) in non-small cell lung cancer (NSCLC) could be defined as continuation of an active treatment until disease progression(PD) in patients who have demonstrated at least a non-progressing status following the first-line chemotherapy (CT). First-line CT is limited to 4 cycles. Although many arguments supporting MT are presented by Rotschild S et al. in this issue of memo [1], a lot of controversies are still surrounding this topic.

Searching the international guidelines for the actual status of MT "rewards" us with a confounding picture. The ASCO guideline definitely does not recommend MT [2], ESMO gives us an undetermined recommendation [3], whereas the NCCN guideline makes clear recommendation for various maintenance strategies and specific agents [4]. For some agents the approval process of the regulatory authority was also confusing. The FDA Oncologic Drugs Advisory Committee voted 12-to-1 against the use of erlotinib as MT due to the modest overall survival (OS) benefit in the SATURN trial [5]. In spite of the vote, FDA decided to expand the indication for erlotinib in this setting. Surprisingly, the FDA approval covers the indication of maintenance erlotinib for patients who previously responded to the first-line chemotherapy, for whom the benefit is virtually absent $(\mathrm{HR}=0.94, p=0.61)[6]$.

Zooming in the design of the MT trials, some major drawbacks can be identified. In the switch maintenance studies, only the non-progressing patients following 4 induction cycles were included. This predefined number of cycles was based on the results of a few randomized trials which showed no improvement in OS for patients treated with 3-4 CT cycles compared with those receiving a longer CT duration $[7,8]$. However, none of these trials addressed the more specific question of whether patients who are responding to chemotherapy, may benefit from treatment beyond 3-4 cycles. As such, we may doubt about the optimal duration of induction therapy in responding patients for whom 6 cycles might be more appropriate, as recommended by the actual guidelines

Correspondence: Mircea Dediu, MD, PhD, Medical Oncology Department, Institute of Oncology Bucharest, Sos Fundeni 252, 022328 Bucharest, Romania.

E-mail: mdediu@clicknet.ro
$[2,3]$. Some supporting data of this bias are emerging from the IFCT-GFPC 0502 study [9]. Patients with advanced NSCLC were started on cisplatin/gemcitabine. The non-progressing patients following 4 cycles were randomized to either observation, continuous maintenance with gemcitabine, or switch maintenance with erlotinib. The best results in terms of improved progression free survival (PFS) were recorded in the gemcitabine continuation arm $(\mathrm{HR}=0.55, p<0.0001)$, while for the erlotinib switch maintenance arm the benefit was less (HR $=0.82, p=0.002)$. Furthermore, the greatest benefit with gemcitabine maintenance was observed in patients with an objective response to first-line therapy as opposed to subjects with stable disease (SD) (HR: $0.44 v s$. 0.68). These data suggest that the full benefits of first-line therapy have not necessarily been achieved after 4 CT cycles. Another criticism of the switch maintenance trial is the insufficient crossover of patients in the non-interventional arm to subsequent therapy following PD. In the JMEN and SATURN trials less than $20 \%$ of the patients in the placebo arms were crossed-over to the drug used as MT at the time of progression $[5,10]$. This bias seems to be supported by the results of the IFCT-GFPC study, which mandates pemetrexed administration in all 3 arms after progression. Despite being positive for PFS, no benefit in survival for any maintenance arm was recorded [9]. Another important observation on this issue came out from the Fidias's study, which randomized the non-progressing patients after induction CT for immediate $v s$. delayed docetaxel [11]. Overall survival of patients in the delayed arm who actually received docetaxel $(62 \%)$ was 12.5 months which was identical to the OS observed in the immediate docetaxel arm. These data underscore the controversy of timing $v$ s. access to second-line therapy.

Although many aspects are still debated, MT is here to be applied, and the clinician is facing the process of patient selection. MT is not suitable for all patient population. Two major conditions make the patient eligible for this strategy: a non-progressing status after 4 CT cycles and an ECOG performance status $0-1$. This accounts for approximately $50 \%$ of the whole patient population $[5,9,11]$. Patients with an altered PS may not benefit from MT, despite having no tumour progression [12]. The tumour biology, reflected by the initial response 
to CT, seems to impact on the type of MT. For instance, maintenance erlotinib is more effective in patients who have SD after first-line CT as opposed to responding patients who virtually derive no benefit [6]. By contrast, continuation maintenance with gemcitabine is more effective in patients who have an initial response as compared with patients with SD [9].

The EGFR mutational status was hoped to provide a reliable predictive marker for the benefit with erlotinib. For the unselected population the benefit of erlotinib in the maintenance setting is modest, in terms of OS ( $12 v s .11$ months) [5]. The most impressive PFS benefit was noted in patients with EGFR mutations (44.6 vs. 13.0 months). This accounts for approximately $3 \%$ of the initial population. Paradoxically, the OS improvement was less in this category $(\mathrm{HR}=0.83)$ compared with the patients with wild type EGFR $(\mathrm{HR}=0.77)$ [13].

One may conclude that we are facing the painful birth of a new concept in the management of advanced NSCLC. Some data are contradictory, some other are more relevant. Many controversies are already here, some others are still to come. For now, MT should be reserved for a selected category of patients and should not be applied as a one-size-fits-all approach. Clearly we need more informative data regarding patient selection and choice of therapy. The additional toxicity inevitably associated with the MT should be balanced against the expected clinical benefit, keeping in mind the clinical profile of the patient. In the daily practice we currently see elderly patients, with associated comorbidities and residual toxicity after first-line C.T. For some of those, who successfully went through the stressful first-line $\mathrm{CT}$, a treatment holiday within a rigorous follow-up program, associated with a more consistent medical involvement in the palliative care may still stand as the best alternative $[3,14]$.

\section{Conflicts of interest}

Mircea Dediu served as a consultant and advisory board member for Sanofi-Aventis, Eli Lilly, and Roche.

\section{References}

[1] Rothschild SI, Gautschi O. Maintenece therapy for patients with advanced non-small celll lung cancer: who to treat and how to treat? memo, 4: 19-22, 2011.
[2] Azzoli C, Baker S, Temin S, et al. Guideline update on chemotherapy for stage IV non-small-cell lung cancer. J Clin Oncol, 27: 6251-6266, 2009.

[3] D'Addario G, Fruh M, Reck M, et al. Metastatic non-small-cell lung cancer: ESMO clinical practice guidelines for diagnosis, treatment and follow up. Ann Oncol, 21: v116-v119, 2010.

4] NCCN Clinical Practice Guidelines in Oncology (NCCN Guidelines) Non-small Cell Lung Cancer. Version 3.2011. Available at: http:// www.nccn.org/professionals/physician_gls/pdf/nscl.pdf. Accessed January 23, 2011.

[5] Cappuzzo F, Ciuleanu T, Stelmakh L, et al. Erlotinib as maintenance treatment in advanced non-small-cell lung cancer: a multicentre, randomised, placebo-controlled phase 3 study. Lancet Oncol, 11: 521-529, 2010.

[6] Cappuzzo F, Ciuleanu T, Park K, Wu Y, et al. Giaccone G. Erlotinib maintenance therapy in patients with stable disease after first-line platinum-doublet chemotherapy for advanced NSCLC. Ann Oncol, 21(Suppl. 8): 369PD, 2010.

[7] Socinski MA, Schell MJ, Peterman A, et al. Phase III trial comparing a defined duration of therapy versus continuous therapy followed by second-line therapy in advanced-stage IIIB/IV non-small-cell lung cancer. J Clin Oncol, 20: 1335-1343, 2002.

[8] Smith IE, O'Brien ME, Talbot DC, et al. Duration of chemotherapy in advanced non-small-cell lung cancer: a randomized trial of three versus six courses of mitomycin, vinblastine, and cisplatin. J Clin Oncol, 19: 1336-1343, 2001.

[9] Perol M, Chouaid C, Milleron BJ, et al. Maintenance with either gemcitabine or erlotinib versus observation with predefined second-line treatment after cisplatin-gemcitabine induction chemotherapy in advanced NSCLC: IFCT-GFPC 0502 phase III study. J Clin Oncol, 28 : 15s, 2010 (suppl; abstr 7507).

[10] Ciuleanu T, Brodowicz T, Zielinski C, et al. Maintenance pemetrexed plus best supportive care versus placebo plus best supportive care for non-small-cell lung cancer: a randomized, double-blind, phase 3 study. Lancet, 374: 1432-1440, 2009.

[11] Fidias PM, Dakhil SR, Lyss AP, et al. Phase III study of immediate compared with delayed docetaxel after front-line therapy with gemcitabine plus carboplatin in advanced non-small-cell lung cancer. J Clin Oncol, 27: 591-598, 2009.

[12] Belani C, Waterhouse D, Ghazal H, et al. Phase III study of maintenance gemcitabine (G) and best supportive care (BSC) versus BSC following standard combination therapy with gemcitabine-carboplatin $(\mathrm{G}-\mathrm{Cb})$ for patients with advanced non-small cell lung cancer (NSCLC). J Clin Oncol, 28: 15s, 2010 (suppl; abstr 7506).

[13] Brugger W, Kim J, Hansen O, et al. Molecular markers and clinica outcome with erlotinib: results from the phase III placebo-controlled SATURN study of maintenance therapy for advanced NSCLC (abstr B9.1). 13th World Conference on Lung Cancer, San Francisco. Available from: http://www.2009worldlungcancer.org/. (Accessed January 29, 2011)

[14] Temel JS, Greer J, Gallagher E, et al. Effect of early palliative care (PC) on quality of life (QOL), aggressive care at the end-of-life (EOL), and survival in stage IV NSCLC patients: Results of a phase III randomized trial. J Clin Oncol, 28: 15s, 2010 (suppl; abstr 7509). 